Arq. Bras. Med. Vet. Zootec., v.68, n.2, p.387-396, 2016

\title{
Efeitos de hormônios esteroides de contraceptivos orais combinados sobre os parâmetros comportamentais de Betta splendens (Regan, 1909)
}

\author{
[Effects of steroid hormones from combined oral contraceptives on the behavioral and physiological \\ parameters of Betta splendens (Regan, 1909)] \\ B.D. Santos ${ }^{1}$, M.C.G. Silva ${ }^{1}$, T.P. Santos ${ }^{1}$, S.C.B.L. Silva ${ }^{1}$, \\ M.R.S. Cadena ${ }^{2}$, P.G. Cadena ${ }^{1 *}$ \\ ${ }^{1}$ Universidade Federal Rural de Pernambuco - Dois Irmãos - Recife, PE \\ ${ }^{2}$ Universidade Federal Rural de Pernambuco - Serra Talhada, PE
}

\begin{abstract}
RESUMO
Os hormônios esteroides presentes em várias gerações de contraceptivos orais combinados (COC) podem se apresentar como disruptores endócrinos, produzindo alterações no comportamento e na fisiologia de peixes. Diante disso, o objetivo deste trabalho foi avaliar os efeitos de hormônios esteroides presentes em COC sobre os parâmetros comportamentais de Betta splendens, um peixe ornamental usado na aquariofilia e bastante agressivo. Machos adultos foram observados pelo método ad libitum para confecção do etograma e divididos em cinco grupos, controle e expostos aos hormônios: 17 $\beta$-estradiol (E2); levonorgestrel e etinilestradiol (LEA - segunda geração de COC); gestodeno e etinilestradiol (GEA - terceira geração de COC); e drospirenona e etinilestradiol (DEA - quarta geração de COC). Os peixes foram expostos por 30 dias à concentração final de $10 \mathrm{ng} / \mathrm{L}$. Foram avaliados os comportamentos pelos métodos de varredura instantânea e animal-focal, bem como o consumo de ração. No método varredura, o comportamento descansar apresentou o maior valor $(54,4 \pm 10,1 \%)$ no grupo E2 $(\mathrm{P}<0,05)$. Os comportamentos agressivos de carga $(16,1 \pm 3,6 \%)$ e recuar e carga $(16,4 \pm 5,1 \%)$ apresentaram os maiores valores no grupo controle em relação aos demais grupos $(\mathrm{P}<0,05)$. Os animais do grupo E2 apresentaram maior frequência de comportamentos inativos $\mathbf{( 7 6 , 1 \% )}$ comparados aos de outros grupos. O comportamento atípico natação errática não foi observado no grupo controle, mas foi observado nos grupos experimentais. Os grupos E2, LEA, GEA e DEA apresentaram redução nos comportamentos agressivos (10\%) quando comparados ao grupo controle pelo método animal-focal. Não foram observadas diferenças na exibição desses comportamentos ao se compararem os animais expostos às diferentes gerações de contraceptivos e no consumo de ração. Pode-se concluir que $17 \beta$-estradiol causou mais efeitos aos peixes e que diferentes gerações de COC apresentaram efeitos tóxicos semelhantes em relação aos comportamentos observados.
\end{abstract}

Palavras-chave: Betta splendens, estradiol, etinilestradiol, etograma, comportamento agressivo

\begin{abstract}
The steroid hormones present in many generations of combined oral contraceptives (COC) can act as endocrine disruptors inducing changes in the behavior and physiology of fish. In this context, the aim of this work was to evaluate the effects of steroid hormones present in COC on behavioral parameters of Betta splendes, an aggressive ornamental fish used in the aquariophily. Adult males were observed with the ad libitum method to develop an ethogram and were divided into five groups, Control and exposed to hormones: 17 $\beta$-estradiol (E2), levonorgestrel and ethinylestradiol (LEA - $2^{\text {nd }}$ COC generation), gestodene and ethinylestradiol (GEA - $3^{\text {rd }}$ COC generation) and drospirenone and ethinylestradiol (DEA - $4^{\text {th }}$ COC Generation). Fish were exposed for 30 days to a final concentration of $10 \mathrm{ng} / \mathrm{L}$. The behavior was evaluated by scan sampling and animal-focal methods, and feed intake. In the scan sampling method,
\end{abstract}

Recebido em 10 de abril de 2015

Aceito em 25 de setembro de 2015

*Autor para correspondência (corresponding author)

E-mail: pabyton.cadena@ufrpe.br 
the Resting behavior showed the highest value $(54.4 \pm 10.1 \%)$ in E2 group $(P<0.05)$. The aggressive

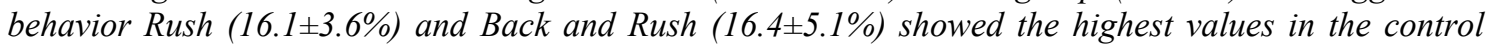
group, compared to the other groups $(P<0.05)$. Animals in the E2 group showed higher frequency of inactive behaviors (76.1\%) compared to other groups. Furthermore, the atypical behavior Erratic swimming was not observed in the control group, but it was observed in the experimental groups. The E2, LEA, GEA and DEA groups showed reduction in aggressive behavior (10\%) compared to the control group by the animal-focal method. Moreover, no difference was observed in the exhibition of these behaviors and feed intake comparing animals exposed to the different generations of contraceptives. It can be concluded that 17ß-estradiol has caused more effects on fish and different generations of COC showed similar toxic effects in the observed behaviors.

Keywords: betta splendens, estradiol, ethinylestradiol, ethogram, aggressive behavior

\section{INTRODUÇÃO}

A presença de diversas substâncias químicas tóxicas nos ecossistemas aquáticos e seus efeitos adversos sobre o sistema endócrino no organismo vêm despertando interesse da comunidade científica. Entre essas substâncias químicas, Frye et al. (2012) descreveram os disruptores endócrinos como agentes que mimetizam, antagonizam ou alteram de forma endógena os níveis de esteroides via mudança nas taxas de síntese ou ação no receptor alvo. Também podem alterar comportamentos associados ou não à reprodução. Esses produtos químicos exercem seus efeitos mesmo em concentrações muito baixas (Dzieweczynski e Buckman, 2013), na ordem de ng/L. Entre os disruptores endócrinos, o $17 \beta$-estradiol (E2), um hormônio sexual feminino derivado do colesterol, tem sido detectado em concentrações ecologicamente relevantes em águas de todo o mundo (Dzieweczynski e Buckman, 2013). Hormônios esteroides e seus derivados podem ser utilizados como contraceptivos orais combinados (COC), sendo produzidos desde 1960 (Stanczyk e Archer, 2014). As progestinas utilizadas nos contraceptivos orais podem ser de quatro tipos. As progestinas da primeira geração são derivadas da testosterona e da progesterona. As de segunda geração são derivadas da 19nortestosterona, e as de terceira geração são derivadas do levonorgestrel. Na quarta geração, é usada a drospirenona, que é um análogo da espironolactona (Orlando e Ellestad, 2014).

Devido ao descarte de hormônios esteroides naturais e sintéticos em ambiente aquático, e ao fato de estes apresentarem atividade mesmo em baixas concentrações, faz-se necessário o estudo desses efeitos na biota aquática. Portanto, os peixes tornaram-se interessantes modelos biológicos para análises toxicológicas. Em razão de as alterações comportamentais serem primeiramente visualizadas (Snowdon, 1999), o estudo do comportamento animal se apresenta como uma excelente ferramenta para essas análises.

Por ser um peixe de fácil aquisição e manejo, além de possuir uma agressividade natural, Betta splendens (Regan, 1909) foi escolhido como modelo animal para o presente trabalho. Betta splendens é conhecido como beta ou peixe de briga, por apresentar comportamento agressivo contra machos da sua espécie, um peixe asiático, criado como peixe ornamental no Brasil, que possui hábito alimentar carnívoro (Fosse et al., 2013). Estudos anteriores com hormônios como disruptores endócrinos já foram realizados utilizando B. splendens como modelo biológico, conforme relatado por Dzieweczynski e Buckman (2013) e Forette et al. (2015).

Devido à importância de mais estudos sobre os efeitos dos hormônios esteroides como disruptores endócrinos no ambiente aquático, bem como de um maior conhecimento dos comportamentos exibidos pela espécie $B$. splendens, o objetivo deste trabalho foi avaliar os efeitos dos hormônios esteroides presentes em três gerações de contraceptivos orais combinados sobre os parâmetros comportamentais de $B$. splendens. Este trabalho visa contribuir para a necessidade de implantação de medidas mitigadoras para reduzir a ação dos disruptores endócrinos no ambiente.

\section{MATERIAL E MÉTODOS}

Os experimentos foram realizados no Laboratório de Ecofisiologia e Comportamento Animal - LECA, da Universidade Federal Rural 
de Pernambuco - UFRPE. Todos os protocolos utilizados foram aprovados pelo Comitê de Ética, processo 001/2014. Machos adultos de Betta splendens foram obtidos de um criadouro comercial em Recife-PE, Brasil. No laboratório, os animais foram aclimatados durante cinco dias, sendo acondicionados em aquários individuais, em sistema semiestático, com capacidade de $1,5 \mathrm{~L}$, à temperatura ambiente $\left(25^{\circ} \mathrm{C}\right)$ e com ciclo de luz natural. A água foi tratada com tiossulfato de sódio para a redução dos níveis de cloro, e o pH mantido em 6,8 durante todo o experimento. Os animais foram alimentados uma vez por dia, com ração comercial extrusada $(40 \%$ de proteína bruta), durante todo o experimento, e foi realizada a lavagem completa dos reservatórios a cada semana. O 17ß-estradiol foi obtido da SIGMA (Lote SLBG0383V), e os três contraceptivos orais combinados foram adquiridos em farmácias da cidade de Recife, PE, Brasil.

Para construção do etograma e observação dos comportamentos, cinco animais controle foram observados durante cinco dias a uma distância preestabelecida $(2,5 \mathrm{~m})$, de modo a não influenciar na exibição dos comportamentos. Foi utilizado o método ad libitum para a identificação dos principais comportamentos exibidos pela espécie, conforme descrito por Cunha Neto (2011), e para a construção do etograma. Nos testes experimentais, os animais foram observados pelo método de varredura instantânea - scan sampling (Altmann, 1974) diariamente por 30 minutos, em intervalos de um minuto de observação e um minuto de descanso. Os comportamentos foram divididos nas categorias locomoção e alimentação. Para a categoria comportamento agressivo, foi utilizado o método animal-focal (Altmann, 1974), com o auxílio de um espelho para estimular o peixe, com duração de seis minutos em intervalos de um minuto de observação e um minuto de descanso. O método de varredura instantânea foi utilizado para as observações dos parâmetros comportamentais no âmbito populacional, enquanto o método animal-focal foi usado para observar todas as ações específicas de um indivíduo; nesse caso, foram analisados os comportamentos agressivos de um indivíduo por vez, como indicado por Altmann (1974).

Para os experimentos de exposição química, $B$. splendens foram divididos em cinco grupos de cinco animais sendo expostos continuamente à adição de hormônios à água (renovação semanal) e um grupo controle. Os tratamentos químicos incluíram o 17 $\beta$-estradiol (grupo E2) puro e contraceptivos orais contendo os seguintes hormônios: i. grupo LEA - progestina da segunda geração: levonorgestrel $(0,015 \mathrm{mg})$ associado ao etinilestradiol $(0,03 \mathrm{mg})$; ii. grupo GEA - progestina da terceira geração: gestodeno $(0,075 \mathrm{mg})$ associado ao etinilestradiol $(0,020 \mathrm{mg})$; e iii. grupo DEA - progestina da quarta geração: drospirenona $(3,0 \mathrm{mg})$ associado ao etinilestradiol $(0,02 \mathrm{mg})$. Os hormônios foram adicionados à água na concentração final de 10ng/L, sendo essa concentração obtida mediante o maceramento de comprimidos, seguido de diluições em álcool e água destilada. Esse valor de concentração foi escolhido baseado nos trabalhos de Dzieweczynski e Buckman (2013) e Clotfelter e Rodriguez (2006). Os animais foram expostos continuamente aos resíduos hormonais por um período de 30 dias; nesse intervalo, o comportamento deles foi observado pelos métodos de varredura instantânea e animal-focal. Finalmente, observou-se o consumo de ração dos animais diariamente pela diferença entre a ração fornecida $(0,8 \mathrm{~g})$ e a consumida. Os resultados foram expressos na forma de média e desviopadrão. Os comportamentos de todos os grupos foram comparados por análise de variância simples (One way ANOVA). Quando a diferença foi significativa, as médias foram comparadas utilizando-se o teste de Tukey com $\mathrm{P}<0,05$, por meio do software Origin Pro 2015 Academic (Origin Lab Corporation, EUA).

\section{RESULTADOS}

Após observação dos comportamentos dos machos adultos pelo método ad libitum conforme descrição de Cunha Neto (2011), foi construído um etograma, com os comportamentos subdivididos nas categorias: Alimentação, Locomoção e Comportamento Agressivo, sumarizados na Tab. 1. 
Tabela 1. Etograma para machos da espécie Betta splendens

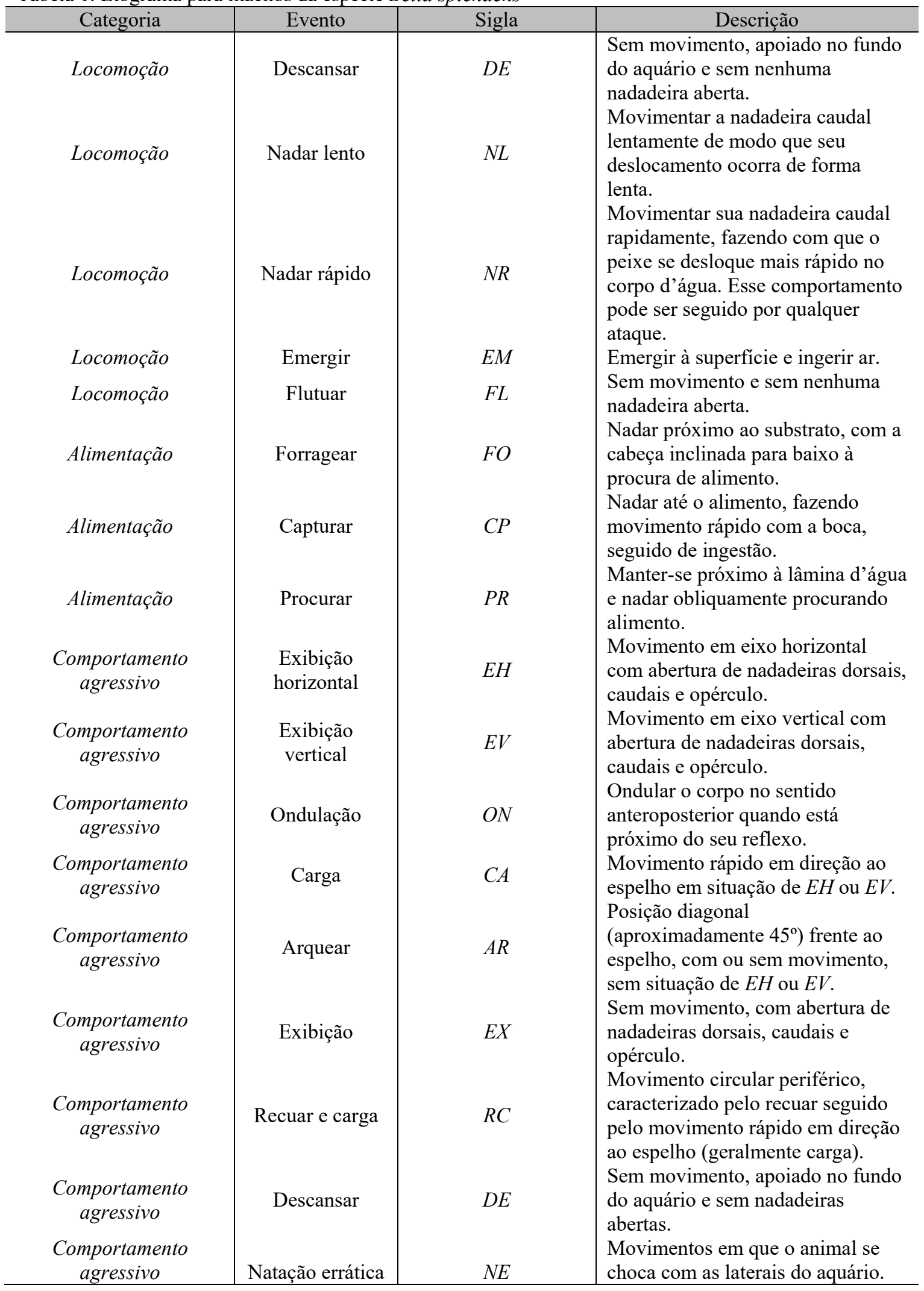


$\mathrm{Na}$ análise por varredura instantânea (Fig. 1A) com cinco animais do grupo controle, foram observados oito comportamentos, divididos em três categorias. No tocante à categoria Locomoção, foram encontrados os comportamentos: $D E(30,3 \pm 22,8 \%)$, o mais exibido; $N L \quad(23,7 \pm 3,8 \%)$, o segundo mais exibido; e também o $E M(19,0 \pm 8,9 \%)$, que é um comportamento característico da espécie, a qual possui o órgão labirinto que a possibilita respirar ar atmosférico; $F L(9,3 \pm 3,8 \%)$ e $N R(2,0 \pm 1,7 \%)$ foram observados com menos frequência. $\mathrm{Na}$ categoria Alimentação, foram exibidos os comportamentos: $F O(11,7 \pm 10,1 \%), \quad C P$ $(2,7 \pm 2,9 \%)$ e $P R(1,3 \pm 1,1 \%)$. A baixa frequência desses atos é justificável pelo fato de os animais serem alimentados até a saciedade, não sendo necessário que eles procurem alimento. Os animais decorreram $60,4 \%$ do tempo exibindo os comportamentos ativos $N L, N R, E M, F O, C P$, $P R$ e $39,6 \%$ do tempo executando os comportamentos inativos $D E$ e $F L$.
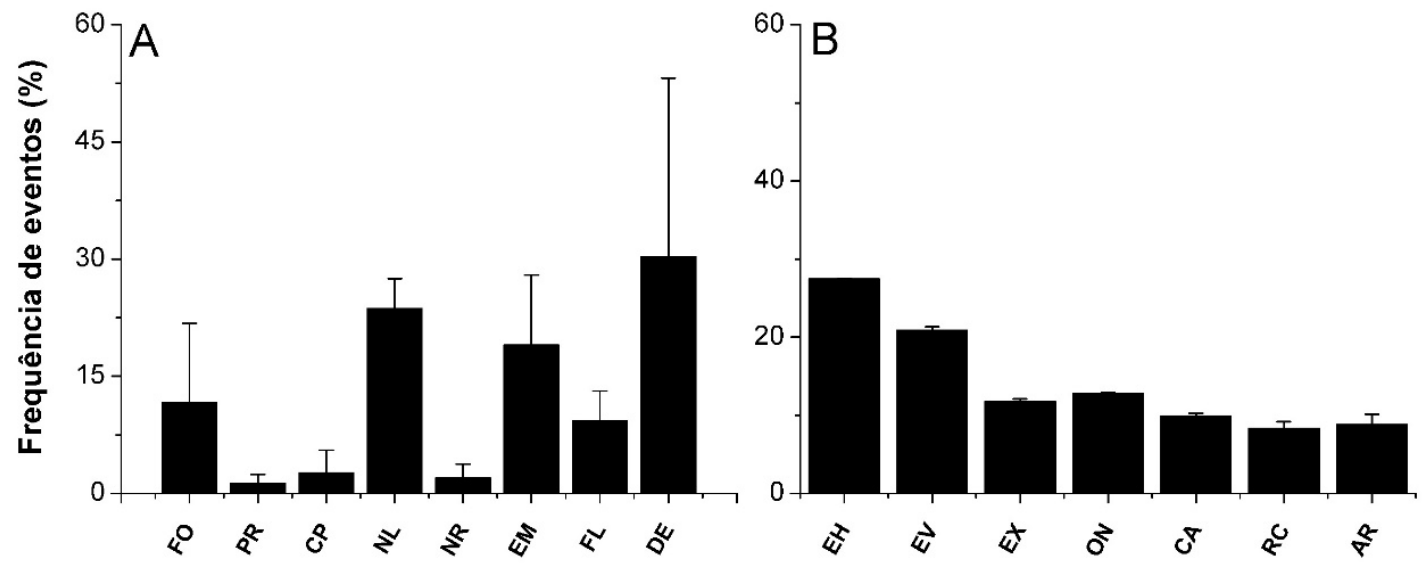

Figura 1. Etograma dos ensaios com os animais adultos de $B$. splendens em cinco dias pelos métodos varredura (A) e focal (B). Siglas: $F O=$ forragear; $P R=$ procurar; $C P=$ capturar; $N L=$ nadar lento; $N R=$ nadar rápido; $E M=$ emergir; $F L=$ flutuar; $D E=$ descansar; $E H=$ exibição horizontal; $E V=$ exibição vertical; $E X=$ exibição; $O N=$ ondulação; $C A=$ carga; $R C=$ recuar e carga; $A R=$ arquear.

$\mathrm{Na}$ análise pelo método animal-focal (Fig. 1B) com cinco animais do grupo controle, foram observados sete comportamentos relacionados à agressividade devido à exposição dos animais ao seu reflexo no espelho. $E H(27,5 \pm 0,1 \%), E V$ $(20,9 \pm 0,5 \%), O N(12,8 \pm 0,1 \%)$ e $E X(11,8 \pm 0,3 \%)$ foram os comportamentos mais comuns observados nos animais. No $C A(9,9 \pm 0,4 \%)$, os animais exibiram tentativas de agredir o seu reflexo devido a aproximações rápidas em direção ao espelho. No comportamento $A R$ $(8,8 \pm 1,4 \%)$, o animal ficou imóvel em frente ao espelho, sem exibir $E H$ ou $E V$, ou seja, sem exibir comportamentos agressivos. Também foi observado o comportamento $R C(8,3 \pm 0,8)$, em que o animal, ao mesmo tempo em que recuava, por se sentir ameaçado pelo seu reflexo, depois rapidamente executava tentativas de ataque.

Os efeitos da exposição química foram avaliados em relação ao comportamento não agressivo pelo método de varredura instantânea (Fig. 2) com os cinco grupos experimentais. $\mathrm{Na}$ categoria Alimentação, não foi observada diferença significativa $(\mathrm{P}<0,05)$ no comportamento $F O$ entre os grupos estudados. Na categoria Locomoção, foram exibidos cinco comportamentos (Fig. 2). No comportamento $D E$, o grupo E2 apresentou a maior frequência $(54,4 \pm 10,1 \%)$, sendo diferente dos demais grupos. Os menores valores para 0 comportamento $D E$ foram observados nos grupos DEA $(20,3 \pm 16,1 \%), \quad$ controle $(19,6 \pm 10,5 \%)$, LEA $(12,8 \pm 10,5 \%)$ e GEA $(13,1 \pm 21,2 \%)$. No comportamento $F L$, o grupo E2 apresentou o menor valor $(21,7 \pm 8,7 \%)$, sendo diferente dos demais grupos. Estes últimos tiveram um aumento na exibição desse comportamento em relação ao grupo E2. Os maiores valores para o comportamento $F L$ foram observados nos grupos LEA $(48,5 \pm 10,6 \%)$, GEA $(48,5 \pm 20,1 \%)$ controle $(46,0 \pm 22,2 \%)$ e DEA $(42,6 \pm 12,8 \%)$, não havendo diferença entre eles. Os comportamentos $D E$ e $F L$, em que os animais 
ficaram imóveis, são considerados inativos. Assim, os animais do grupo E2 apresentaram mais da metade $(54,4 \%)$ da frequência dos comportamentos exibindo o comportamento $D E$. Em relação aos grupos com exposição aos contraceptivos, não foram observadas diferenças significativas quanto aos comportamentos $D E$ e $F L$ entre eles.

Nos comportamentos $N R$ e $N L$, o grupo E2 apresentou os menores valores $(3,6 \pm 2,2 \%$; $9,0 \pm 5,6 \%$, respectivamente). No comportamento $N R$, foi observado que o grupo E2 apresentou a menor frequência desse comportamento e foi diferente do grupo LEA $(10,0 \pm 4,7 \%)$, que apresentou a maior frequência. Contudo, não houve diferenças do grupo E2 em relação aos demais grupos: controle $(6,0 \pm 3,9 \%)$, GEA $(6,8 \pm 4,3 \%)$ e DEA $(5,9 \pm 3,1 \%)$. Os grupos com animais expostos a contraceptivos orais não apresentaram diferenças significativas entre eles. No comportamento $N L$, novamente o grupo E2 apresentou a menor frequência e foi diferente dos grupos controle $(21,4 \pm 10,3 \%), \quad$ GEA $(24,2 \pm 9,1 \%)$ e DEA $(22,0 \pm 4,4 \%)$. Diante desses resultados, observou-se que os animais do grupo E2 tinham maior frequência de comportamentos inativos comparados aos de outros grupos. Também foi observado que não houve diferença na exibição desses comportamentos ao se compararem os animais expostos às diferentes gerações de contraceptivos.

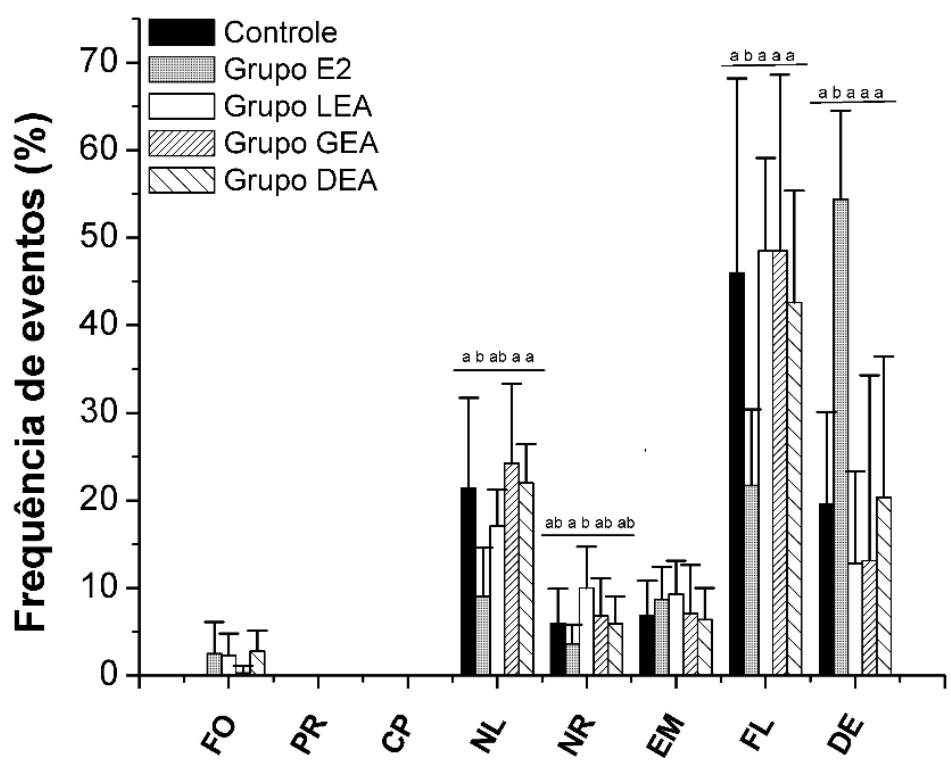

Figura 2. Etograma dos ensaios com os animais adultos de B. splendens expostos aos resíduos hormonais por 30 dias pelo método varredura. Siglas: $F O=$ forragear; $P R=$ procurar; $C P=$ capturar; $N L=$ nadar lento; $N R=$ nadar rápido; $E M=$ emergir; $F L=$ flutuar; $D E=$ descansar; grupo $\mathrm{E} 2=17 \beta$-estradiol; grupo LEA = levonorgestrel associado ao etinilestradiol; grupo GEA = gestodeno associado ao etinilestradiol; grupo DEA = drospirenona associado ao etinilestradiol. Letras iguais não diferem estatisticamente pelo teste de Tukey, com $\mathrm{P}<0,05$.

Os resultados da exposição química, em relação ao comportamento agressivo, são apresentados na Fig. 3. Foi observado que, na categoria Comportamento Agressivo, houve diferença significativa nos grupos estudados $(\mathrm{P}<0,05)$. Para $E H$, os grupos GEA $(24,0 \pm 2,8 \%)$ e E2 $(22,7 \pm 4,0 \%)$ apresentaram os maiores valores para esse comportamento, sendo diferentes dos grupos controle $(17,8 \pm 3,6 \%)$, DEA $(19,7 \pm 1,1 \%)$ e LEA $(14,7 \pm 1,7 \%)$. No comportamento $E V$, os maiores valores foram observados nos grupos controle $(29,6 \pm 7,5 \%)$, GEA $(24,9 \pm 2,6 \%)$ e E2 $(23,6 \pm 5,2 \%)$, não existindo diferenças entre eles. Os grupos DEA $(16,4 \pm 2,9 \%)$ e LEA $(16,0 \pm 4,1 \%)$ apresentaram os menores valores, não havendo diferenças entre eles. Não foi observada diferença significativa $(\mathrm{P}>0,05)$ para $\mathrm{o}$ comportamento $E X$ entre todos os grupos estudados. No comportamento $O N$, foi observado que o grupo DEA $(5,9 \pm 3,0 \%)$ apresentou os maiores valores em relação aos demais grupos. Os grupos LEA $(1,4 \pm 1,0 \%)$ e E2 $(0,6 \pm 0,9 \%)$ 
apresentaram os menores valores para este comportamento. O grupo controle apresentou os maiores valores para os comportamentos $C A$ $(16,1 \pm 3,6 \%)$ e $R C(16,4 \pm 5,1 \%)$, sendo diferente de todos os grupos experimentais. Estes últimos tiveram redução na exibição dos dois comportamentos agressivos: GEA $(6,6 \pm 2,9 \%$; $3,6 \pm 3,1 \%)$, LEA $(5,9 \pm 3,2 \% ; 5,2 \pm 1,7 \%)$ e DEA $(5,7 \pm 2,9 \% ; \quad 5,0 \pm 1,7 \%)$. Foi observada uma redução significativa $\quad(\mathrm{P}<0,05) \quad$ dos comportamentos $C A$ e $R C$ nos grupos experimentais, independentemente da geração dos COC utilizados, em comparação ao grupo controle.

O grupo LEA apresentou o maior valor para o comportamento $A R(31,5 \pm 4,2 \%)$, sendo diferente de todos os outros grupos. No grupo controle, foi observado o menor valor $(4,1 \pm 8.9 \%)$ para o comportamento $A R$, sendo diferente estatisticamente $(\mathrm{P}<0,05)$ de todos os grupos experimentais. $A R$ é um comportamento inativo, em que o animal fica imóvel, flutuando, sem exibir comportamento agressivo, e, portanto, os grupos expostos a resíduos hormonais apresentaram aumento comparados ao grupo controle. Não foi observado o comportamento
$D E$ no grupo controle. Já os grupos LEA $(4,7 \pm 3,0 \%)$ e DEA $(8,6 \pm 4,4 \%)$ apresentaram os maiores valores para o comportamento $D E$, não sendo diferentes entre eles, mas diferentes dos grupos E2 e controle. O comportamento $D E$ também indica inatividade do animal. $A R$ e $D E$ são comportamentos inativos, em que o animal não exibe comportamento agressivo; estes comportamentos não foram observados no grupo controle. $\mathrm{O}$ comportamento $N E$ também não foi observado no grupo controle. Os grupos GEA $(2,7 \pm 2,6 \%)$, DEA $(3,4 \pm 2,1 \%)$ e LEA $(4,2 \pm 0,6 \%)$ foram diferentes estatisticamente em relação ao grupo controle, mas não foram diferentes entre si. Este comportamento não é comumente exibido pelo animal, sendo um efeito colateral provocado pela presença de agentes estressores, como, por exemplo, os resíduos hormonais presentes nos $\mathrm{COC}$ adicionados à agua.

Não foram observadas diferenças significativas $(\mathrm{P}>0,05)$ em todos os grupos estudados em relação ao consumo de ração. Os animais consumiram rapidamente toda a quantidade da ração extrusada, $0,80 \mathrm{~g}$ para cada animal, que era fornecida diariamente.

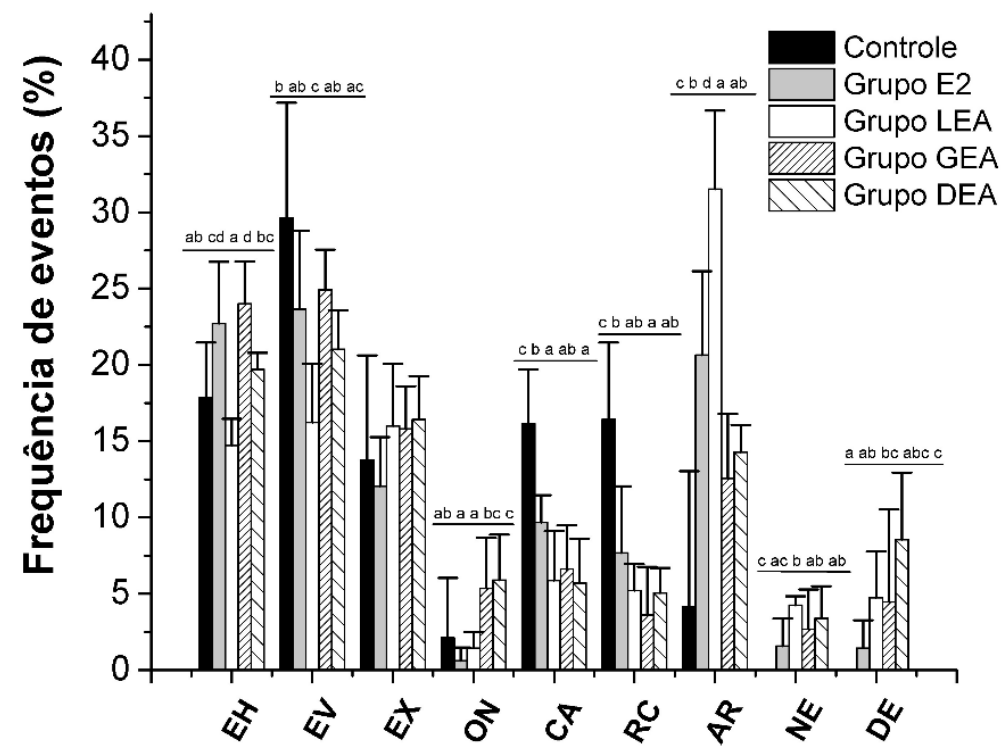

Figura 3. Etograma dos ensaios com os animais adultos de B. splendens expostos aos resíduos hormonais por 30 dias pelo método focal. Siglas: $E H=$ exibição horizontal; $E V=$ exibição vertical; $E X=$ exibição; $O N=$ ondulação; $C A=$ carga; $R C=$ recuar e carga; $A R=$ arquear; $N E=$ natação errática; grupo E2 $=17 \beta-$ estradiol; grupo LEA = levonorgestrel associado ao etinilestradiol; grupo GEA = gestodeno associado ao etinilestradiol; grupo DEA = drospirenona associado ao etinilestradiol. Letras iguais não diferem estatisticamente pelo teste de Tukey, com $\mathrm{P}<0,05$. 


\section{DISCUSSÃO}

Os resultados indicaram que resíduos hormonais presentes nos COC interferiram no comportamento animal, bem como na exibição dos comportamentos agressivos, estimulados pelo espelho dos machos adultos de $B$. splendens comparados aos animais do grupo controle. $\mathrm{O}$ grupo controle também apresentou maior frequência de comportamentos agressivos e ausência dos comportamentos $N E$ e $D E$ que são associados ao estresse. Na literatura (Clotfelter e Rodriguez, 2006), foi observado que tanto a exposição aguda quanto a crônica a disruptores endócrinos podem afetar o comportamento e a fisiologia de peixes machos que vivem em ambientes que contenham a presença desses compostos tóxicos, como o E2 e o $17 \alpha-$ etinilestradiol. Comumente, tem sido demonstrado que o comportamento pode ser um bom indicador em estudos de fisiologia (Snowdon, 1999). A vantagem em usar parâmetros comportamentais deu-se pelo fato de ser um método não invasivo, de baixo custo e com facilidade para a obtenção de grande quantidade de dados.

Com relação ao 17ß-estradiol (E2), foi observado que o grupo E2 passou a maior parte do tempo exibindo o comportamento inativo $D E$ no método varredura, além de apresentar uma diminuição dos comportamentos agressivos, sendo o grupo que mais teve modificações nas frequências dos comportamentos em relação ao grupo controle. Esse resultado comprova o relatado por Clotfelter e Rodriguez (2006), os quais observaram que a exposição ao $17 \beta$ estradiol, em várias concentrações, reduziu o comportamento agressivo de $B$. splendens machos quando medido pela sua resposta ao reflexo no espelho. Esses autores sugerem que tal efeito ocorre pela redução nos níveis de andrógenos nos peixes machos (Bell, 2001). Montello e Todd (2010) também observaram, em seu estudo com fêmeas de B. splendens, que o E2 modificou os comportamentos agressivos e os não agressivos após elas terem sido expostas à solução estoque de $5 \mathrm{mg} / \mu \mathrm{L}$ de hormônio durante 28 dias. Os mesmos autores observaram que as fêmeas ficaram mais letárgicas quando expostas ao hormônio, indicando que a exposição a quantidades ambientais baixas pode ter um efeito significativo no comportamento agressivo dessa espécie.
Com relação ao $17 \alpha$-etinilestradiol, foi observado que, embora os grupos experimentais LEA, GEA e DEA contenham, além deste, progestinas de gerações diferentes, eles não diferem estatisticamente entre si $(\mathrm{P}>0,05)$ na maioria dos comportamentos estudados. Porém, houve diferenças em relação ao grupo controle. Esses grupos foram os que menos exibiram os comportamentos mais agressivos $(C A$ e $R C)$ pelo método animal-focal. Dzieweczynski e Buckman (2013) observaram os efeitos do $17 \alpha-$ etinilestradiol em $B$. splendens no âmbito populacional dentro de uma rede de indivíduos, e seus resultados demonstraram que a exposição aguda a $15 \mathrm{ng} / \mathrm{L}$ de $17 \alpha$-etinilestradiol diminuiu a capacidade dos machos em se comunicarem com vários indivíduos simultaneamente, bem como os tipos de mudanças comportamentais que normalmente ocorrem quando outro indivíduo está presente; estas também foram reduzidas ou não estavam presentes nos machos expostos. Forette et al. (2015) observaram os efeitos do $17 \alpha$-etinilestradiol $(10 \mathrm{ng} / \mathrm{L})$ sobre a interação entre machos e fêmeas de $B$. splendens $\mathrm{e}$ verificaram uma redução na interação entre eles. Portanto, o $17 \alpha$-etinilestradiol não só reduziu o comportamento agressivo, mas pode também interferir no sucesso reprodutivo do animal. $\mathrm{O}$ $17 \alpha$-etinilestradiol pode ser comumente encontrado em águas de superfície em concentração de 4 - 12ng/L (Forette et al., 2015) devido ao grande uso de COC, o que aumenta a probabilidade de a biota aquática ter contato com esse composto tóxico (Frye et al., 2012). Trabalhos que contenham efeitos tóxicos de levonorgestrel, gestodeno e drospirenona no comportamento de peixes são escassos na literatura. O levonorgestrel e o gestodeno levaram a uma redução nos níveis de testosterona e E2 em peixes fêmeas, e o gestodeno a um aumento nos níveis de testosterona em peixes machos de Pimephales promelas (Orlando e Ellestad, 2014). O levonorgestrel tem ação androgênica em peixes teleósteos, apresentando efeitos tóxicos em fêmeas, como a masculinização e a redução na ovulação (Overturf e Huggett, 2015). Overturf e Huggett (2015) avaliaram o efeito tóxico do levonorgestrel $(125 \mathrm{ng} / \mathrm{L})$ em larvas de Pimephales promelas por 28 dias. Foi observada uma redução na transcrição de RNAm para a produção do hormônio folículo estimulante (FSH) e de enzimas esteroidogênicas, após sete dias de exposição. Modificações na atividade do 
eixo pituitária adrenal podem interferir na produção de hormônios androgênicos. Tal efeito, somado aos efeitos tóxicos do $17 \alpha-$ etinilestradiol, poderia justificar a redução no comportamento agressivo do grupo LEA. Em relação à drospirenona, Zucchi et al. (2014) observaram os efeitos tóxicos da drospirenona isolada (55 - 5442ng/L) e em associação com a progesterona em fêmeas de Danio rerio. Esses autores encontraram alterações no ritmo circadiano e na atividade de receptores de hormônios esteroides. Diante disso, sabe-se que a drospirenona pode modificar a atividade de hormônios esteroides, o que poderia justificar as modificações observadas no comportamento agressivo do grupo DEA. Adicionalmente, os grupos expostos aos hormônios esteroides apresentaram o comportamento atípico $N E$, não observado no grupo controle. Esse comportamento sugere que houve uma alteração na forma de deslocamento dos animais, causada pela ação dos disruptores endócrinos.

Além dos comportamentos, também foi avaliado o consumo de ração. Foi observado que, durante todo o experimento, os animais se alimentaram normalmente, não havendo diferença estatística $(\mathrm{P}<0,05)$ entre todos os grupos experimentais.

\section{CONCLUSÃO}

Ao se avaliarem os efeitos dos hormônios sexuais femininos associados às progestinas contidas nos COC sobre os parâmetros comportamentais dos machos de Betta splendens, pôde-se observar que ocorreu uma diminuição dos comportamentos ativos e agressivos em todos os grupos expostos aos resíduos hormonais. Essa redução foi mais pronunciada no grupo tratado com o 17 $\beta$-estradiol, o que demonstra que esse hormônio causou mais efeitos aos B. splendens machos. As diferentes gerações de $\mathrm{COC}$ apresentaram efeitos tóxicos semelhantes em relação aos comportamentos observados, e foram observados comportamentos atípicos, como a natação errática. Diante do exposto, mesmo com o avanço da tecnologia farmacêutica melhorando as propriedades farmacológicas das diferentes gerações de contraceptivos testados, seus efeitos tóxicos em $B$. splendens não foram reduzidos em relação aos parâmetros estudados.

\section{AGRADECIMENTOS}

À Universidade Federal Rural de Pernambuco, por permitir o desenvolvimento deste projeto; ao CNPq, pelo apoio financeiro (Processo 477215/2013-0).

\section{REFERÊNCIAS}

ALTMANN, J. Observational study of behavior: sampling methods. Behaviour, v.49, p.227-267, 1974.

BELL, A.M. Effects of an endocrine disrupter on courtship and aggressive behaviour of male three-spined stickleback, Gasterosteus aculeatus. Anim. Behav., v.62, p.775-780, 2001.

ClOTFELTER, E.D.; RODRIGUEZ, A.C. Behavioral changes in fish exposed to phytoestrogens. Environ. Pollut., v.144, p.833839, 2006.

CUNHA NETO, J.S. Dissociação entre observação e interação na modificação do display agressivo do Betta splendens. 2011. $51 \mathrm{f}$. Dissertação (Mestrado em Teoria e Pesquisa do Comportamento) - Núcleo de Teoria e Pesquisa do Comportamento, Universidade Federal do Pará, Belém, PA.

DZIEWECZYNSKI, T.L.; BUCKMAN, C.M. Acute exposure to 17alpha-ethinylestradiol disrupts audience effects on male-male interactions in Siamese fighting fish, Betta splendens. Horm. Behav., v.63, p.497-502, 2013.

FORETTE, L.M.; MANNION, K.L.; DZIEWECZYNSKI, T.L. Acute exposure to 17alpha-ethinylestradiol disrupts audience effect on male-female interactions in Betta_splendens. Behav. Process., v.113, p.172-8, 2015.

FOSSE, P.J.; MATTOS, D.C.; CARDOSO, L.D. et al. Estratégia de coalimentação na sobrevivência e no crescimento de larvas de Betta splendens durante a transição alimentar. Arq. Bras. Med. Vet. Zootec., v.65, p.1801-1807, 2013.

FRYE, C.A.; BO, E.; CALAMANDREI, G. et al. Endocrine disrupters: a review of some sources, effects, and mechanisms of actions on behaviour and neuroendocrine systems. $J$. Neuroendocrinol., v.24, p.144-159, 2012. 
MONTELLO, M.; TODD, N.E. The Potential for Disruption of Aggressive Behavior in Female Betta splendens by Environmental Estrogens. $J$. Behav. Neurosci. Res., v.8, p.26-34, 2010.

ORLANDO, E.F.; ELLESTAD, L. E. Sources, concentrations, and exposure effects of environmental gestagens on fish and other aquatic wildlife, with an emphasis on reproduction. Gen. Comp. Endocrinol., v.203, p.241-249, 2014.

OVERTURF, M.D.; HUGGETT, D.B. Responses to various exposure durations of levonorgestrel during early-life stages of fathead minnows (Pimephales promelas). Aquat. Toxicol., v.161, p.33-40, 2015.
SNOWDON, T.C. O significado da pesquisa em Comportamento Animal. Estud. Psicol., v.4, p.365-373, 1999.

STANCZYK, F.Z.; ARCHER, D.F. Gestodene: a review of its pharmacology, potency and tolerability in combined contraceptive preparations. Contraception, v.89, p.242-252, 2014.

ZUCCHI, S.; MIRBAHAI, L.; CASTIGLIONI, $\mathrm{S}$. et al. Transcriptional and physiological responses induced by binary mixtures of drospirenone and progesterone in zebrafish (Danio rerio). Environ. Sci. Technol., v.48, p.3523-31, 2014. 